Ophthalmologe $2022 \cdot 119: 962-965$ https://doi.org/10.1007/s00347-021-01499-x Eingegangen: 17. August 2021 Überarbeitet: 23. August 2021 Angenommen: 24. August 2021 Online publiziert: 17 . September 2021 ๑ Der/die Autor(en) 2021

\section{Okuläre Argyrose nach langjähriger Applikation von Wimpern- und Augenbrauenfarbe}

\author{
Marlene Saßmannshausen ${ }^{1,2} \cdot$ Martina C. Herwig-Carl ${ }^{1,2} \cdot$ Frank G. Holz . \\ Karin U. Loeffler ${ }^{1,2}$ \\ ${ }^{1}$ Klinik für Augenheilkunde, Universitätsklinikum Bonn, Bonn, Deutschland \\ ${ }^{2}$ Sektion Ophthalmopathologie, Universitätsklinikum Bonn, Bonn, Deutschland
}

\section{Anamnese}

Eine 69-jährige Frau stellte sich zur Mitbeurteilung einer diffusen Bindehautpigmentierung beidseits vor, die im Rahmen einer augenärztlichen Routineuntersuchung festgestellt wurde. In den zurückliegenden 10 Jahren fanden keine augenärztlichen Konsultationen statt. Akute ophthalmologische Beschwerden oder eine Sehminderung wurden von der Patientin verneint. Allgemeinanamnestisch seien ein Schilddrüsenknoten und eine rechtsseitige Karotisstenose unter aktuell medikamentöser Behandlung mit Aspirin, Statinen und Ezetimib bekannt. Der bestkorrigierte Visus betrug beidseits 1,0, der Augeninnendruck 17/15 mm Hg. Vorangegangene okuläre Eingriffe, u.a. Laserbehandlungen oder Operationen, wurden verneint. Eine zusätzliche Einnahme von Nahrungsergänzungsmitteln lag nicht vor.

\section{Befund}

Spaltlampenmikroskopisch zeigte sich beidseits eine diffuse schwärzliche Bindehautpigmentierung insbesondere im inferioren Bereich, aber auch mit Beteiligung von Plica und Karunkel bei gleichzeitiger Aussparung der subtarsalen Bindehaut des Ober- und Unterlids (- Abb. 1). Darüber hinaus zeigte sich beidseits seitengleich ein diffuser, punktförmiger, braungrauer Hornhautbeschlag, der sich in den tieferen Hornhautschichten gleichmäßig von der zentralen Hornhaut bis hin zum Horn- hautlimbus erstreckte. Es zeigte sich kein Anhalt für eine akute oder abgelaufene intraokulare Entzündung, und die Linse und der Fundus waren altersentsprechend. In der erweiterten Anamnese berichtete die Patientin, seit ca. 30 Jahren 1- bis 2-mal wöchentlich aus kosmetischen Gründen eine schwarz färbende Augenbrauenund Wimpernfarbe (Swiss O Par, Fa. Rufin cosmetic, Deutschland) aufzutragen. Berufsanamnestisch war die Patientin als Sacharbeiterin im öffentlichen Dienst beschäftigt, eine langfristige erhöhte Exposition gegenüber chemischen Produkten war nicht bekannt. Aufgrund des unklaren okulären Befundes und zum Ausschluss einer malignen Genese entschieden wir uns für eine Probeexzision im Bereich der Bindehautpigmentierung.

\section{Histopathologische Untersuchung}

Für die histopathologische Routineuntersuchung wurden die entnommenen Bindehautproben in 4\%igem Paraformaldehyd fixiert und histologisch aufgearbeitet.

In der histologischen Untersuchung des Bindehautbiopsats ( $\mathbf{0} \mathbf{A b b}$. 2a) zeigte sich in der Hämatoxylin-Eosin Färbung (HE) ein regelrechtes becherzellhaltiges Bindehautepithel mit einer diffusen stromalen Entzündungsreaktion. In der stärkeren Vergrößerung (- Abb. 2b) imponierte eine vorwiegend im oberen Stroma gelegene feingranuläre, schwärzliche Pigmentierung, die vorwiegend an Kollagenfasern angelagert erschien und nur selten Zellen zugeordnet werden konnte. Stellenweise 

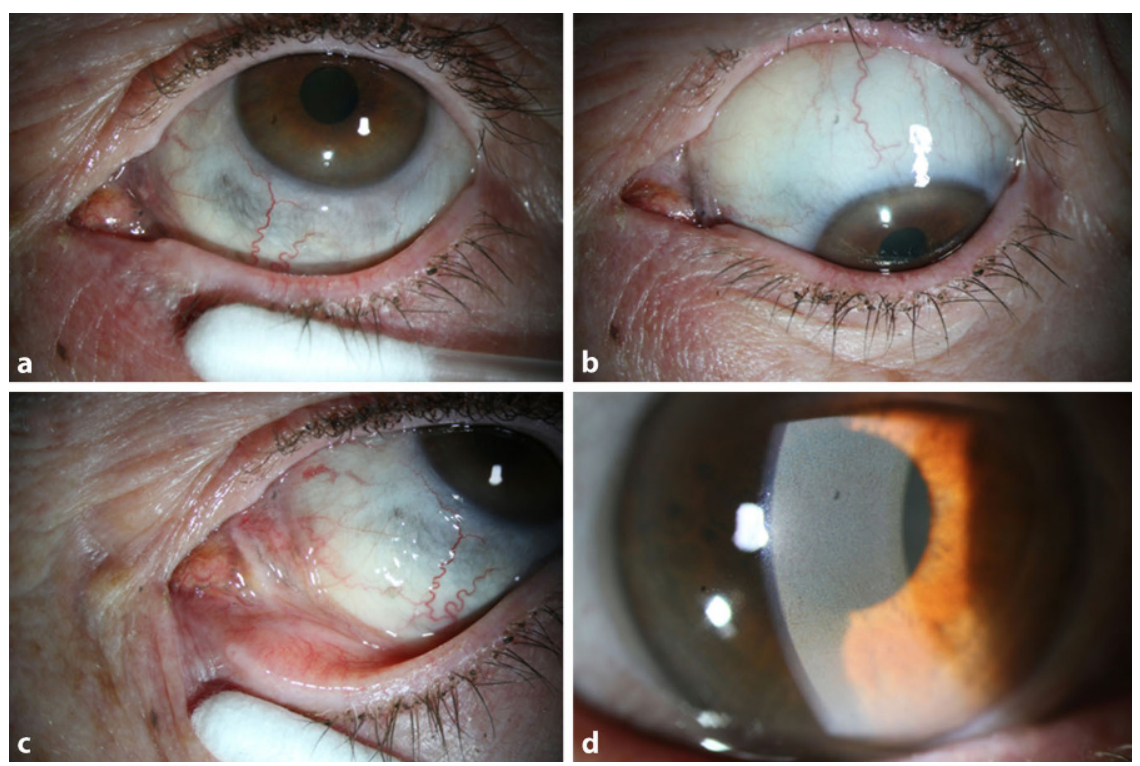

Abb. 1 ॥ Spaltbilddokumentation des Vorderabschnittsbefundes mit prominenter Pigmentierung der Bindehaut sowie Beteiligung der Plica und Karunkel unter Aussparung des subtarsalen Bereichs (a-c). Braungrauer Beschlag vermutlich in der Descemet-Membran ohne Hinweis auf einen intraokularen Reiz (d)

zeigte sich die entsprechende Pigmentierung allerdings auch in basalen Epithelzellen. Die Spezialfärbung (MassonFontana) zum Nachweis von Silber bestätigte den Ursprung der Granula als silberhaltig (- Abb. 3).

\section{Diagnose und Verlauf}

In der Zusammenschau des klinischen und histopathologischen Befundes ließ sich die Diagnose von Silbereinlagerungen im Bereich der Bindehaut nach jahrzehntelanger Applikation der Wimpern- und Augenbrauenfarbe stellen. Naheliegend ist, dass auch die in der klinischen Untersuchung gut sichtbaren Ablagerungen der Hornhautrückfläche auf die Applikation des mit Silbernitrat versetzten Kosmetikaprodukts zurückzuführen sind. Eine ergänzende serologische Untersuchung zum Nachweis einer systemischen Silbernitratbelastung ergab einen unauffälligen Befund. Die weiterführende Diagnostik inklusive Vorderabschnitts-OCT, Pentacam und Endothelzellzahlmessung ergab einen zum jetzigen Zeitpunkt unauffälligen Vorderabschnittsbefund ohne Hinweis auf eine Endotheldekompensation. In den Verlaufsuntersuchungen 4 und 12 Wochen nach der Probenentnahme präsentierte sich der Befund lokal unverändert bei gleichbleibend vol- ler Sehschärfe trotz sofortigen Absetzens des Kosmetikaprodukts nach der Probenentnahme.

\section{Diskussion}

Neben dem bekannten Einsatz von Silber in Metallprodukten oder auch Schmuckartikeln wird Silber häufig in kosmetischen Produkten in Form von Mikrosilber eingesetzt. Zudem handelt es sich aufgrund seiner antibakteriellen und auch entzündungshemmenden Charakteristika um einen häufigen Bestandteil in Hautpflegeprodukten, Deodorants und auch Kosmetika. Bei Neugeborenen wurden früher zudem silberhaltige Augentropfen als Prophylaxe einer Gonokokkenkonjunktivitis eingesetzt (sog. Credé-Prophylaxe).

In einer Reihe von Fallberichten wurden Silberreste in Haut und Schleimhaut, aber auch in inneren Organen nach der systemischer Einnahme von silberhaltigen Medikamenten in oraler Form nachgewiesen $[1,11]$. Auch ophthalmologisch wurde bereits von Silberresten im Bereich der Augenadnexe, der Bindehaut und Hornhaut (im Bereich der Descemet-Membran) sowie auch intraokular u.a. im Kammerwinkel nach längerfristiger topischer oder systemischer Einnahme von silberhaltigen Medikamenten oder auch der beruflichen
Exposition gegenüber Silber berichtet [2, 4, 6-10]. Eine zunehmende Anzahl von Fallberichten thematisiert den ophthalmologischen Nachweis von Silberresten nach der langjährigen lokalen Anwendung von silberhaltigen Augentropfen oder Kosmetikaprodukten wie Wimpern- und Augenbrauenfärbemitteln [3, 4]. In diesem Zusammenhang konnte bereits histologisch der Nachweis von abgelagerten Silberresten im Tränensackbereich oder auch der Bindehaut nach Applikation von silberhaltigen Augentropfen erbracht werden [3,9].

Ähnlich wie in unserem beschriebenen Fall wurde in einer Fallserie von Gallardo et al. nach langjähriger Kosmetikapplikation eine gräulich-braune Ablagerung im Bereich des Hornhautstromas und der Descemet-Membran beobachtet [3]. Ein histologischer Nachweis fehlte hier jedoch, da kein Hornhautmaterial in vivo entnommen werden konnte. In 2 weiteren Arbeiten, die ein entnommenes Hornhautpräparat mittels Elektronenmikroskopie untersuchten $[5,8]$, sowie auch in weiteren Arbeiten, die In-vivo-Untersuchungen u.a. mit der konfokalen Mikroskopie durchführten [6, 12], konnten darüber hinaus Silberreste im Bereich der Bowman-Schicht, in dem Hornhautstroma und der Descemet-Membran nach der langjährigen Exposition gegenüber Silber nachgewiesen werden. Interessanterweise wurde im Rahmen einer 3-jährigen Nachverfolgung eines Patienten zwar ein Rückgang der Einlagerungen im Stroma beobachtet, jedoch persistierten weiterhin Silberreste im Bereich der Descemet-Membran bei gleichzeitig abnehmender Anzahl an regelrecht konfigurierten Endothelzellen [12].

Unser Bericht beschreibt nun einen weiteren Fall mit hochgradigem Verdacht auf eine descemetale Ablagerung von Silber bei gleichzeitig histologisch nachgewiesener Ablagerung von Silber in der korrespondierenden Bindehaut. Eine histologische Untersuchung des originalen Wimpern- und Augenbrauenfärbepräparats zeigte zudem dasselbe morphologische Bild wie die Bindehautprobe. So ist zu vermuten, dass es über den Tränenfilm zu einer jahrzehntelangen Exposition der Hornhaut mit Silbernitrat gekommen ist wobei noch hinzukommt, dass das Produkt häufiger als empfohlen angewendet wurde. Obwohl der genaue Pathomecha- 


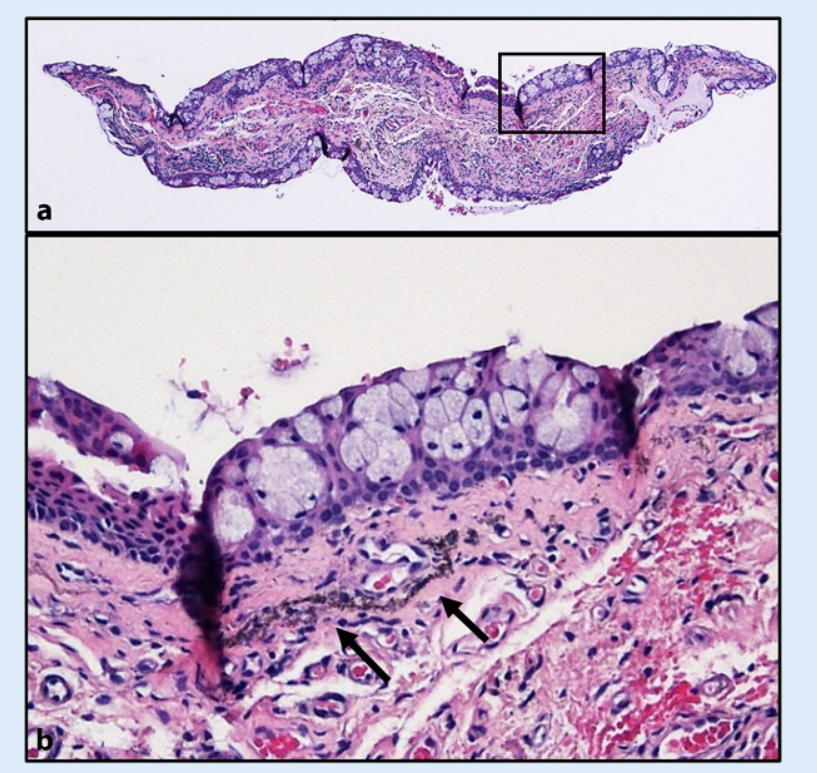

Abb. 2 \ Histologische Übersichtsaufnahme des Bindehautpräparats (a, Hämatoxylin-Eosin[HE]-Färbung, Vergr. 40:1). Bei höherer Vergrößerung zeigen sich ein regelrechtes becherzellhaltiges Bindehautepithel und eine braun-schwärzliche Pigmentierung im Stroma (Pfeile, HE-Färbung, Vergr. 100:1)

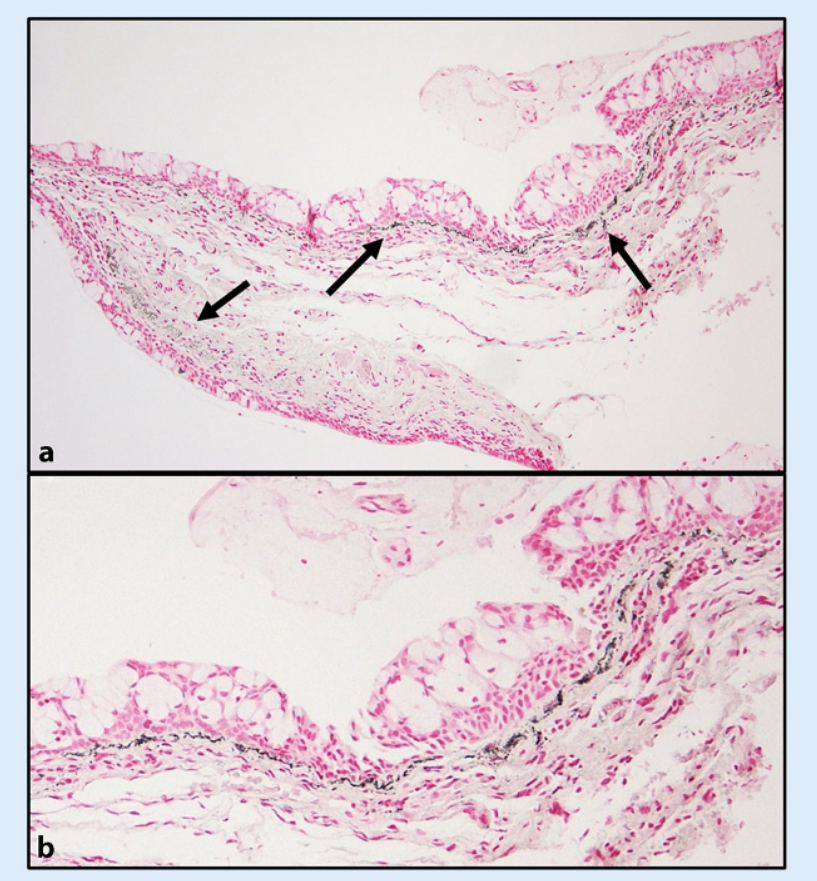

Abb. $3 \Delta$ In der Silberfärbung (Masson-Fontana) zeigt sich zur HE-Färbung korrelierend der positive Nachweis von silberhaltigen Granula im Bereich des Bindehautstromas (Pfeile, a, Vergr. 40:1). In der höheren Vergrößerung ist die vorwiegend stromale Ablagerung gut nachvollziehbar (b, Vergr. 100:1) nismus unter Berücksichtigung der aktuellen Literatur unklar bleibt, ist es denkbar, dass das Silbernitrat über Diffusion oder auch einen aktiven Transport vom Epithel bis zur Descemet-Membran gelangt. In der Verlaufskontrolle unserer Patientin war keine Abnahme der Endothelzahl messbar, und auch die weiterführenden Vorderabschnittsuntersuchungen mittels OCT, Endothelzellmessung und PentacamDiagnostik waren unauffällig. Auch wenn wir hier von einer descemetalen Silberablagerung ausgehen, bleibt in unserem Fall zu beobachten, ob im langfristigen Verlauf eine Endotheldekompensation oder andere Komplikationen auftreten.

\section{Fazit für die Praxis}

- Die Anwendung von silberhaltigen Wimpern- und Augenbrauenfärbemitteln kann zu einer konjunktivalen und auch kornealen Ablagerung von Silber führen.

- Klinisch und histologisch sind diese von anderen pigmentierten Prozessen abzugrenzen.

- Komplikationen sind zwar selten, können jedoch langfristig nicht ausgeschlossen werden.

\section{Korrespondenzadresse}

\section{Dr. med. Marlene Saßmannshausen}

Klinik für Augenheilkunde, Universitätsklinikum Bonn

Ernst-Abbe-Str. 2, 53127 Bonn, Deutschland marlene.sassmannshausen@ukbonn.de

Funding. Open Access funding enabled and organized by Projekt DEAL.

\section{Einhaltung ethischer Richtlinien}

Interessenkonflikt. M. Saßmannshausen reports grants from Bonfor Gerok Stipendium (0-137.0030) der medizinischen Fakultät der Universität Bonn, outside the submitted work., M.C. Herwig-Carl reports grants from Deutsche Forschungsgemeinschaft, grants from EPIC-XS.EU, personal fees from Glaxo Smith Kline, outside the submitted work. F.G. Holz reports grants and personal fees from Bayer, grants and personal fees from Novartis, grants from Acucela, grants and personal fees from Apellis, grants and personal fees from Kanghong, grants and personal fees from Roche/Genentech, personal fees from Pixium Vision, grants and personal fees from Allergan, personal fees from $L$ in Bioscience, personal fees from Oxurion, grants from NightStar X, grants from Bioequ/ Formycon, personal fees from Stealth Therapeutics, personal fees from Kodiak, grants, personal fees and non-financial support from Geuder, personal fees from Grayburg Vision, personal fees from iveric bio, grants, personal fees and non-financial support from Heidelberg Engineering, non-financial support from Centervue, personal fees and non-financial support from Zeiss, personal fees and non-financial support from Optos, outside the submitted work. K.U. Loeffler gibt an, dass kein Interessenkonflikt besteht.

Für diesen Beitrag wurden von den Autoren keine Studien an Menschen oder Tieren durchgeführt. Für die aufgeführten Studien gelten die jeweils dort angegebenen ethischen Richtlinien. Für Bildmaterial oder anderweitige Angaben innerhalb des Manuskripts, über die Patienten zu identifizieren sind, liegt von ihnen und/oder ihren gesetzlichen Vertretern eine schriftliche Einwilligung vor.

Open Access. Dieser Artikel wird unter der Creative Commons Namensnennung 4.0 International Lizenz veröffentlicht, welche die Nutzung, Vervielfältigung, Bearbeitung, Verbreitung und Wiedergabe in jeglichem Medium und Format erlaubt, sofern Sie den/die ursprünglichen Autor(en) und die Quelle ordnungsgemäß nennen, einen Link zur Creative Commons Lizenz beifügen und angeben, ob Änderungen vorgenommen wurden.

Die in diesem Artikel enthaltenen Bilder und sonstiges Drittmaterial unterliegen ebenfalls der genannten Creative Commons Lizenz, sofern sich aus der Abbildungslegende nichts anderes ergibt. Sofern das betreffende Material nicht unter der genannten Creative Commons Lizenz steht und die betreffende Handlung nicht nach gesetzlichen Vorschriften erlaubt ist, ist für 
die oben aufgeführten Weiterverwendungen des Materials die Einwilligung des jeweiligen Rechteinhabers einzuholen.

Weitere Details zur Lizenz entnehmen Sie bitte der Lizenzinformation auf http://creativecommons.org/ licenses/by/4.0/deed.de.

\section{Literatur}

1. Claessens D, Zeitz PF, Beckers H (2020) Blaugraue Verfärbung von Haut und Bindehaut. Ophthalmologe 117(1):66-68. https://doi.org/10.1007/ s00347-019-0899-x

2. Flögel W, Widmeier S, Hotz P, Schärer L, Barthelmes D, Landau K, Thiel MA (2006) Corneale und conjunctivale Befunde bei systemischer Argyrose. Klin Monbl Augenheilkd 223(5):390-392. https:// doi.org/10.1055/s-2006-926556

3. Gallardo MJ, Randleman JB, Price KM, Johnson DA, Acosta S, Grossniklaus HE, Stulting RD (2006) Ocular argyrosis after long-term self-application of eyelash tint. Am J Ophthalmol 141(1):198-200. https://doi.org/10.1016/j.ajo.2005.07.054

4. Gutman FA, Crosswell HH (1968) Argyrosis of the cornea without clinical conjunctival involvement. Am J Ophthalmol 65(2):183-187. https://doi.org/ 10.1016/0002-9394(68)93584-8

5. Hanna C, Fraunfelder FT, Sanchez J (1974) Ultrastructural study of argyrosis of the cornea and conjunctiva. Arch Ophthalmol 92(1):18-22. https://doi.org/10.1001/archopht. 1974.01010010022006

6. He X, Simmons NL, Wozniak RAF (2020) Anterior segment optical coherence tomography in ocular argyrosis. Cornea 39(11):1433-1435. https://doi. org/10.1097/ICO.0000000000002323

7. Kamath $Y$, Sinha A (2013) Ocular argyrosis in a jeweller. BMJ Case Rep. https://doi.org/10.1136/ bcr-2013-009742

8. Karcioglu ZA, Caldwell DR (1985) Corneal argyrosis: histologic, ultrastructural and microanalytic study. Can J Ophthalmol 20(7):257-260

9. Loeffler KU, Lee WR (1987) Argyrosis of the lacrimal sac. Graefes Arch Clin Exp Ophthalmol 225(2):146-150. https://doi.org/10.1007/ BF02160348

10. Stafeeva K, Erlanger M, Velez-Montoya R, Olson JL (2012) Ocular argyrosis secondary to long-term ingestion of silver nitrate salts. Clin Ophthalmol 6:2033-2036. https://doi.org/10.2147/OPTH. S37898

11. Wadhera A, Fung M (2005) Systemic argyria associated with ingestion of colloidal silver. Dermatol Online J11(1):12

12. Wu M, Wang $X$, Shao T, Wang $Y$ (2017) Case report: in vivo confocal microscopic appearance of corneal argyrosis. Optom Vis Sci 94(11):1066-1069. https://doi.org/10.1097/OPX.0000000000001133 